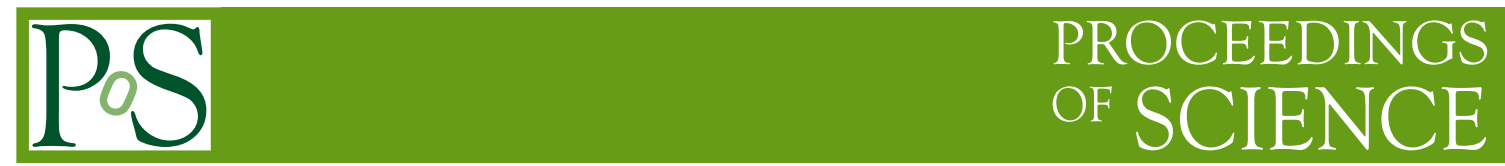

\title{
A new mechanism of Higgs-radion mixing
}

\section{Igor Volobuev*}

Skobeltsyn Institute of Nuclear Physics, Moscow State University

E-mail: volobuev@theory.sinp.msu.ru

We consider a brane world model with the interbrane distance stabilized by a bulk scalar field and assume that this field is coupled to the Higgs field on the negative tension brane. This interaction naturally leads to spontaneous breaking of the SM gauge symmetry and to a Higgs-radion mixing. We estimate this mixing.

The XXth International Workshop High Energy Physics and Quantum Field Theory September 24-October 1, 2011

Sochi Russia

${ }^{*}$ Speaker. 


\section{Introduction}

It is a common knowledge that in QFT fields with the same quantum numbers can mix, if there is an interaction between them. A canonical example is the mixing of the weak hypercharge $U(1)$ gauge filed and the neutral component of the $\mathrm{SU}(2)$ gauge field, which gives rise to the electromagnetic field and the field of the $\mathrm{Z}$ boson. In extensions of the SM additional fields can mix with the fields of the SM, if they have the same quantum numbers.

In the present contribution we consider an extension of the SM based on the Randall-Sundrum model with two branes stabilized by a bulk scalar field [1, 2]. A characteristic feature of this model is the presence of a massive scalar radion field, which describes the fluctuations of the branes with respect to each other. This field has the same quantum numbers as the neutral Higgs field. Thus, the radion field can mix with the Higgs field, if they are coupled.

Originally, a Higgs-radion coupling in the unstabilized Randall-Sundrum model arising due to a curvature term on the brane was put forward in [3]. Then such a coupling and the resulting Higgsradion mixing in the case of the stabilized model were discussed in paper [ $₫$ ] without taking into account the KK tower of higher scalar excitations. In what follows we discuss a model, where such a coupling naturally arises due to a mechanism of spontaneous symmetry breaking on the brane involving the stabilizing scalar field. Unlike the mentioned papers our approach takes into account the influence of the KK tower of higher scalar excitations on the parameters of the Higgs-radion mixing, which turns out to be of importance.

\section{The model}

Let us denote the coordinates in five-dimensional space-time $E=M_{4} \times S^{1} / Z_{2}$ by $\left\{x^{M}\right\} \equiv$ $\left\{x^{\mu}, y\right\}, M=0,1,2,3,4, \mu=0,1,2,3$, the coordinate $x^{4} \equiv y, \quad-L \leq y \leq L$ parameterizing the fifth dimension. It forms the orbifold, which is realized as the circle of the circumference $2 L$ with the points $y$ and $-y$ identified. Correspondingly, the metric $g_{M N}$ and the scalar field $\phi$ satisfy the orbifold symmetry conditions

$$
g_{\mu v}(x,-y)=g_{\mu v}(x, y), g_{\mu 4}(x,-y)=-g_{\mu 4}(x, y), g_{44}(x,-y)=g_{44}(x, y), \phi(x,-y)=\phi(x, y) .
$$

The branes are located at the fixed points of the orbifold, $y=0$ and $y=L$.

The action of the stabilized brane world model can be written as

$$
\begin{aligned}
S & =2 M^{3} \int d^{4} x \int_{-L}^{L} d y R \sqrt{-g}-\int d^{4} x \int_{-L}^{L} d y\left(\frac{1}{2} g^{M N} \partial_{M} \phi \partial_{N} \phi+V(\phi)\right) \sqrt{-g}- \\
& -\int_{y=0} \sqrt{-\tilde{g}} \lambda_{1}(\phi) d^{4} x+\int_{y=L} \sqrt{-\tilde{g}}\left(-\lambda_{2}(\phi)+L_{S M-H P}+L_{i n t}(\phi, h)\right) d^{4} x .
\end{aligned}
$$

Here the signature of the metric $g_{M N}$ is chosen to be $(-,+,+,+,+), g=\operatorname{det} g_{M N}, V(\phi)$ is a bulk scalar field potential and $\lambda_{1,2}(\phi)$ are brane scalar field potentials, $\tilde{g}=\operatorname{det} \tilde{g}_{\mu \nu}$, with $\tilde{g}_{\mu \nu}$ denoting the metric induced on the branes. The Lagragian $L_{S M-H P}$ is the SM Lagrangian without the Higgs potential that is replaced by the interaction Lagrangian

$$
L_{\text {int }}(\phi, h)=-\lambda\left(|h|^{2}-\xi \phi\right)^{2} .
$$


The parameter $\xi$ in this formula has dimension $m^{1 / 2}$ and will be specified later. We would like to note here that one can also take interaction Lagrangian (2.3) with $\phi^{2}$ in the brackets. A similar coupling of the Higgs field to the dilaton field is discussed in [5].

The background solution for the metric and the scalar field, which preserves the Poincare invariance in any four-dimensional subspace $y=$ const, looks like

$$
d s^{2}=e^{-2 A(y)} \eta_{\mu v} d x^{\mu} d x^{v}+d y^{2} \equiv \gamma_{M N}(y) d x^{M} d x^{N}, \quad \phi(x, y)=\phi(y),
$$

$\eta_{\mu \nu}$ denoting the flat Minkowski metric, whereas the background solution for the Higgs field is standard

$$
h=\left(\begin{array}{c}
0 \\
\frac{v}{\sqrt{2}}
\end{array}\right),
$$

all the other SM fields being equal to zero.

If one substitutes this ansatz into the equations of motion corresponding to action (2.2), one gets a relation between the vacuum value of the Higgs field and the value $\phi_{2}$ of the field $\phi$ on the brane at $y=L, \quad \phi_{2}=v^{2} /(2 \xi)$, and a rather complicated system of nonlinear differential equations for functions $A(y), \phi(y)\left(^{\prime} \equiv d / d y\right)$ :

$$
\begin{gathered}
\frac{d V}{d \phi}+\frac{d \lambda_{1}}{d \phi} d(y)+\frac{d \lambda_{2}}{d \phi} d(y-L)=-4 A^{\prime} \phi^{\prime}+\phi^{\prime \prime} \\
12 M^{3}\left(A^{\prime}\right)^{2}+\frac{1}{2}\left(V-\frac{1}{2}\left(\phi^{\prime}\right)^{2}\right)=0 \\
\frac{1}{2}\left(\frac{1}{2}\left(\phi^{\prime}\right)^{2}+V+\lambda_{1} \delta(y)+\lambda_{2} \delta(y-L)\right)=-2 M^{3}\left(-3 A^{\prime \prime}+6\left(A^{\prime}\right)^{2}\right)
\end{gathered}
$$

To find an analytic solution to this system we use the results of papers [1, 6]. Let us consider a special class of potentials, which can be represented as

$$
V(\phi)=\frac{1}{8}\left(\frac{d W}{d \phi}\right)^{2}-\frac{1}{24 M^{3}} W^{2}(\phi) .
$$

It is easy to check that if we put

$$
\phi^{\prime}(y)=\operatorname{sign}(y) \frac{1}{2} \frac{d W}{d \phi}, \quad A^{\prime}(y)=\operatorname{sign}(y) \frac{1}{24 M^{3}} W(\phi),
$$

then equations (2.6) are valid everywhere, except for the branes. In order the equations of motion be valid everywhere, one needs to finetune the brane potentials $\lambda_{1,2}(\phi)$.

Let us take $W(\phi)$ to be

$$
W=24 M^{3} k-u \phi^{2},
$$

so that $V(\phi)$ is a quartic potential. Finetuned potentials on the branes can be chosen as follows:

$$
\lambda_{1}=W(\phi)+\beta_{1}^{2}\left(\phi-\phi_{1}\right)^{2}, \quad \lambda_{2}=-W(\phi)+\beta_{2}^{2}\left(\phi-\phi_{2}\right)^{2} .
$$

The parameters of the potentials $k, u, \phi_{1,2}, \beta_{1,2}$, when made dimensionless by the fundamental fivedimensional energy scale of the theory $M$, should be positive quantities of the order $O(1)$, i.e. there should be no hierarchical difference in the parameters.

For such a choice of the potentials the solution of the equations of motion looks as follows [1]

$$
\phi(y)=\phi_{1} e^{-u|y|}, \quad A(y)=k|y|+\frac{\phi_{1}^{2}}{48 M^{3}} e^{-2 u|y|} .
$$


The interbrane distance is defined by the boundary conditions for the field $\phi$ and is expressed in terms of the parameters of the model by the relation $L=\ln \left(\phi_{1} / \phi_{2}\right) / u$. Thus, we see that the brane separation distance is stabilized.

It turns out that, with the function $A(y)$ given by (2.10), it is impossible to find exact solutions for modes other than the zero one. For this reason in paper [7] an approximation $u L \ll 1$ was put forward, which is rather general and physically interesting. Keeping in $A$ only the terms linear in $y$, we get

$$
A(y)=\tilde{k}|y|, \quad \tilde{k}=k-\frac{\phi_{1}^{2}}{24 M^{3}} u .
$$

Thus, in this approximation the metric of the stabilized model coincides with that of the unstabilized model, where a substitution $k \rightarrow \tilde{k}$ was made. In this case the equations of the model can be solved exactly, and the corresponding formulas for eigenfunctions and eigenvalues were discussed in detail in [8].

Now the linearized theory is obtained by representing the metric, the scalar and the Higgs fields as

$$
\begin{aligned}
g_{M N}(x, y) & =\gamma_{M N}(y)+\frac{1}{\sqrt{2 M^{3}}} h_{M N}(x, y), \quad \phi(x, y)=\phi(y)+\frac{1}{\sqrt{2 M^{3}}} f(x, y), \\
h(x) & =\left(\begin{array}{c}
0 \\
\frac{v+\sigma(x)}{\sqrt{2}}
\end{array}\right)
\end{aligned}
$$

substituting this representation into action (2.2) and keeping the terms of the second order in $h_{M N}$, $f$ and $\sigma$. The resulting Lagrangian is the second variation Lagrangian of the stabilized model found in [2] supplemented by the terms

$$
\left[-\frac{1}{2} \partial_{\mu} \sigma \partial^{\mu} \sigma-\frac{1}{2} 2 \lambda v^{2} \sigma^{2}+\frac{2 \lambda v \xi}{\sqrt{2 M^{3}}} f \sigma-\frac{\lambda \xi^{2}}{2 M^{3}} f^{2}\right] \delta(y-L) .
$$

\section{Higgs-radion mixing}

It is not difficult to find that the part of the Lagrangian relevant to the Higgs-radion mixing is

$$
\left[-\frac{1}{2 M^{3}}\left(\beta_{2}^{2}+\lambda \xi^{2}\right) f^{2}+\frac{2 \lambda \nu \xi}{\sqrt{2 M^{3}}} f \sigma-\frac{1}{2} 2 \lambda v^{2} \sigma^{2}\right] \delta(y-L) .
$$

First let us neglect the interaction of the fields $f$ and $\sigma$. In this case we can, in the standard way, find the mass spectrum and the wave functions of the excitations of the field $f$; they coincide with those found in [胢], if one replaces there $\beta_{2}^{2} \rightarrow \beta_{2}^{2}+\lambda \xi^{2}$, the second term coming from potential (2.3). Then we expand the field $f$ in these modes, substitute this expansion into the second variation Lagrangian and integrate over $y$. As a result, we get a four-dimensional Lagrangian, in which there is an interaction between the modes and the Higgs field coming from the term $2 \lambda \nu \xi f \sigma / \sqrt{2 M^{3}}$.

This term can be transformed as follows. In paper [2] it was shown that there exists the gauge condition $g^{\prime}=\exp (-2 A) \phi^{\prime} f /\left(3 M^{3}\right)$, relating the field $f$ to the scalar field $g=\exp (-2 A) h_{44}$, coming from the metric. Using the mode decomposition of $g(x, y)$ and the boundary condition for the mode wavefunction $g_{n}(y)$ at $y=L$ we have:

$$
\frac{f(L)}{3 M^{3}}=\left.\frac{g^{\prime} e^{2 A}}{\phi^{\prime}}\right|_{y=L}=\left.\frac{g^{\prime}}{\phi^{\prime}}\right|_{y=L}=-\frac{g^{\prime}(L)}{u \phi_{2}}=-\sum_{n=1}^{\infty} \frac{\mu_{n}^{2}}{\left(\beta_{2}^{2}+\lambda \xi^{2}-u\right) u \phi_{2}} g_{n}(L) \phi_{n}(x) .
$$


Thus, the interaction of the modes $\phi_{n}(x)$ with the Higgs field looks like

$$
-\frac{3 M^{3} 2 \lambda v \xi}{\sqrt{2 M^{3}}} \sum_{n=1}^{\infty} \frac{\mu_{n}^{2}}{\left(\beta_{2}^{2}+\lambda \xi^{2}-u\right) u \phi_{2}} g_{n}(L) \phi_{n}(x) \sigma(x) .
$$

As we already mentioned, $\xi$ is a free parameter that can be chosen arbitrarily. But it is more interesting to construct it form the other parameters of the model, since in so doing we spare a free parameter. Here we will pursue this idea and take $\xi=u^{2} / \sqrt{2 M^{3}}$, because this combination of the model parameters has the right dimension and order of magnitude. In this case the term $\lambda \xi^{2}$ in the denominator is negligibly small and can be dropped. Then we get the interaction Lagrangian

$$
-\frac{3 \lambda u v}{\left(\beta_{2}^{2}-u\right) \phi_{2}} \sum_{n=1}^{\infty} \mu_{n}^{2} g_{n}(L) \phi_{n}(x) \sigma(x)
$$

Let us denote

$$
\frac{3 \lambda u v}{\left(\beta_{2}^{2}-u\right) \phi_{2}} \mu_{n}^{2} g_{n}(L)=a_{n}^{2}
$$

If we take the parameters of the model that correspond to the radion mass $\mu_{1}=200 \mathrm{GeV}$ [ []], we approximately have $a_{1}^{2}=2 \lambda(30)^{2} G e V^{2}$, i.e. $a_{1}^{2} / \mu_{1}^{2} \ll 1$, if the Higgs mass also does not exceed $200 \mathrm{GeV}$. Since the boundary values of the wave functions of the modes decrease with $n$, we also have $a_{n}^{2} / \mu_{n}^{2} \ll 1$. Thus, the mass matrix is

$$
\mathscr{M}=\left(\begin{array}{cccccc}
2 \lambda v^{2} & a_{1}^{2} & a_{2}^{2} & \cdots & a_{n}^{2} & \cdots \\
a_{1}^{2} & \mu_{1}^{2} & 0 & \cdots & 0 & \cdots \\
a_{2}^{2} & 0 & \mu_{2}^{2} & \cdots & 0 & \cdots \\
. & . & . & \cdots & . & \cdots \\
. & . & . & \cdots & . & \cdots \\
a_{n}^{2} & 0 & 0 & \cdots & \mu_{n}^{2} & \cdots \\
. & . & . & \cdots & . & \cdots \\
. & . & . & \cdots & . & \cdots
\end{array}\right) .
$$

It is not difficult to find that

$$
\operatorname{det}(\mathscr{M}-t I)=\left(2 \lambda v^{2}-t-\sum_{n=1}^{\infty} \frac{a_{n}^{4}}{\mu_{n}^{2}-t}\right) \Pi\left(\mu_{n}^{2}-t\right) .
$$

Since $a_{n}^{2} / \mu_{n}^{2} \ll 1$ we can expect that the eigenvalues of the matrix are close to $2 \lambda v^{2}$ and $\mu_{n}^{2}$, but the original masses are not the eigenvalues. Then, denoting $\Delta^{2}=\sum_{n=2}^{\infty} \frac{a_{n}^{4}}{\mu_{n}^{2}}$, we approximately get for the Higgs mass

$$
m_{H}^{2}=2 \lambda v^{2}-\Delta^{2}-\frac{\mu_{1}^{2}-2 \lambda v^{2}+\Delta^{2}}{2}\left(\sqrt{1+\frac{4 a_{1}^{4}}{\left(\mu_{1}^{2}-2 \lambda v^{2}+\Delta^{2}\right)^{2}}}-1\right)
$$

and for the radion mass

$$
m_{1}^{2}=\mu_{1}^{2}+\frac{\mu_{1}^{2}-2 \lambda v^{2}+\Delta^{2}}{2}\left(\sqrt{1+\frac{4 a_{1}^{4}}{\left(\mu_{1}^{2}-2 \lambda v^{2}+\Delta^{2}\right)^{2}}}-1\right) .
$$


Thus, we see that if $\mu_{1}^{2} \geq 2 \lambda v^{2}$, this coupling always makes the radion heavier and the Higgs boson lighter. If this is not the case, a special analysis is needed to find out how the masses alter.

The normalized eigenvectors of the matrix $\mathscr{M}$ corresponding to these eigenvalues can be approximately found by the standard technique. We note that the eigenvectors of the scalar modes with $n \geq 2$ are exactly orthogonal to the Higgs and the radion eigenvectors, and orthogonal to each other up to terms of order $\frac{a_{k}^{2}}{\mu_{k}^{2}} \frac{a_{n}^{2}}{\mu_{n}^{2}} \ll 1$. However, in the approximation under consideration the Higgs and the radion eigenvectors are not orthogonal, which is not good. For this reason we find a new approximation for the radion mass that makes the eigenvectors orthogonal. Namely, denoting $\rho=\sum_{n=2}^{\infty} \frac{a_{n}^{4}}{\mu_{n}^{4}}$, we demand that

$$
1+\rho+\frac{a_{1}^{4}}{\left(\mu_{1}^{2}-m_{H}^{2}\right)\left(\mu_{1}^{2}-m_{1}^{2}\right)}=0
$$

which ensures the orthogonality and gives for the radion mass

$$
m_{1}^{2}=\mu_{1}^{2}+\frac{a_{1}^{4}}{\left(\mu_{1}^{2}-m_{H}^{2}\right)(1+\rho)}
$$

a value that does not differ much from the one found above. In what follows we will use this value for the radion mass.

Since the matrix $\mathscr{M}$ is symmetric and real, it is diagonalized by an orthogonal transformation, and the kinetic terms of the mass eigenstate fields are also diagonal. These mass eigenstate fields are expressed in terms of the original fields as

$$
\begin{aligned}
& \tilde{\sigma}=N_{H} \sigma-N_{H} \frac{a_{1}^{2}}{\mu_{1}^{2}-m_{H}^{2}} \phi_{1}-N_{H} \sum_{n=2}^{\infty} \frac{a_{n}^{2}}{\mu_{n}^{2}} \phi_{n} \\
& \tilde{\phi}_{1}=N_{1} \sigma-N_{1} \frac{a_{1}^{2}}{\mu_{1}^{2}-m_{1}^{2}} \phi_{1}-N_{1} \sum_{n=2}^{\infty} \frac{a_{n}^{2}}{\mu_{n}^{2}} \phi_{n} \\
& \tilde{\phi}_{k}=\frac{a_{k}^{2}}{\mu_{k}^{2}} \sigma+\phi_{k}, \quad k \geq 2 .
\end{aligned}
$$

where

$$
N_{H}=\left(1+\rho+\frac{a_{1}^{4}}{\left(\mu_{1}^{2}-m_{H}^{2}\right)^{2}}\right)^{-\frac{1}{2}}, \quad N_{1}=\left(1+\rho+\frac{a_{1}^{4}}{\left(\mu_{1}^{2}-m_{1}^{2}\right)^{2}}\right)^{-\frac{1}{2}} .
$$

The Yukawa couplings of the mass eigenstate fields to fermions look like

$$
\Gamma \bar{\psi} \psi\left(N_{H} \tilde{\sigma}+N_{1} \tilde{\phi}_{1}-\sum_{n=2}^{\infty} \frac{a_{n}^{2}}{\mu_{n}^{2}} \tilde{\phi}_{n}\right)
$$

and to the trace of the energy-momentum tensor

$$
-\frac{1}{\sqrt{32 M^{3}}}\left(C_{0} \tilde{\sigma}+\sum_{n=1}^{\infty} C_{n} \tilde{\phi}_{n}\right) T_{\mu}^{\mu}
$$


where the constants are defined by

$$
\begin{aligned}
& C_{0}=-N_{H} \frac{a_{1}^{2} g_{1}(L)}{\mu_{1}^{2}-m_{H}^{2}}-N_{H} \sum_{n=2}^{\infty} \frac{a_{n}^{2}}{\mu_{n}^{2}} g_{n}(L) \\
& C_{1}=-N_{1} \frac{a_{1}^{2} g_{1}(L)}{\mu_{1}^{2}-m_{1}^{2}}-N_{1} \sum_{n=2}^{\infty} \frac{a_{n}^{2}}{\mu_{n}^{2}} g_{n}(L) \\
& C_{k}=g_{k}(L), \quad k \geq 2 .
\end{aligned}
$$

Thus, we see again that though the interaction of the individual higher excited scalar states may be weak, their cumulative effect on the Higgs-radion mixing may be observable due to their noticeable contributions to the values of parameters $\Delta^{2}$ and $\rho$ and lead to certain changes in the collider phenomenology of the Higgs boson. A similar contribution of the directly unobservable tensor KK modes to scattering processes was discussed in [9], where it was shown that this contribution is of the same order as that of the lowest modes and for this reason should be always taken into account.

\section{Acknowledgments}

The author is grateful to E. Boos and M. Smolyakov for interesting and useful discussions.

\section{References}

[1] O. DeWolfe, D.Z. Freedman, S.S. Gubser, A. Karch, Phys. Rev. D 62 (2000) 046008.

[2] E. E. Boos, Y. S. Mikhailov, M. N. Smolyakov and I. P. Volobuev, Mod. Phys. Lett. A 21 (2006) 1431 [arXiv:hep-th/0511185].

[3] G. F. Giudice, R. Rattazzi and J. D. Wells, hep-ph/0002178.

[4] C. Csaki, M.L. Graesser, G.D. Kribs, Phys. Rev. D 63 (2001) 065002.

[5] F. Bezrukov and D. Gorbunov, JHEP 1005 (2010) 010 [arXiv:0912.0390 [hep-ph]].

D. Gorbunov, "Light inflaton in Bottom decays: a close look at the dawn of the Universe", PoS(QFTHEP2011)063.

[6] A. Brandhuber, K. Sfetsos, JHEP 9910 (1999) 013.

[7] E. E. Boos, Y. S. Mikhailov, M. N. Smolyakov and I. P. Volobuev, Nucl. Phys. B 717 (2005) 19 [arXiv:hep-th/0412204].

[8] E.E. Boos, I.P. Volobuev, Y.A. Kubyshin, M.N. Smolyakov, Theor. Math. Phys. 131 (2002) 629 [arXiv:hep-th/0105304]; Class. Quant. Grav. 19 (2002) 4591.

[9] E. E. Boos, V. E. Bunichev, M. N. Smolyakov and I. P. Volobuev, Phys. Rev. D 79 (2009) 104013 [arXiv:0710.3100 [hep-ph]]. 